14 Costa DL, Dreher KL. Bioavailable transition metals in particulate matter mediate cardiopulmonary injury in healthy and compromised animal models. Environ Health Perspect 1997; 105: Suppl. 5, 1053-1060.

15 Gurgueira SA, Lawrence J, Coull B, et al. Rapid increases in the steady-state concentration of reactive oxygen species in the lungs and heart after particulate air pollution inhalation. Environ Health Perspect 2002; 110: 749-755.

\title{
Fatal acute respiratory distress syndrome with diffuse alveolar damage: donor lymphocyte infusion imputability?
}

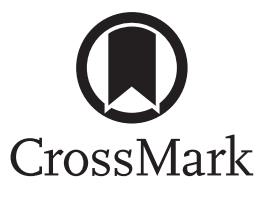

To the Editor:

Donor lymphocyte infusion (DLI) is increasingly used after allogeneic haematopoietic stem cell transplantation (allo-HSCT), as a pre-emptive or curative immunotherapy of relapsed haematological malignancies, enhancing graft-versus-tumour effects [1]. The main complication is graft-versus-host disease (GVHD), with cumulative incidences of $40-60 \%$ and 33-61\% for acute and chronic GVHD, respectively, typically developing 32-42 days after DLI [2]. Pulmonary complications after DLI are poorly documented. To our knowledge, there are no cases of diffuse alveolar damage (DAD) [3] after DLI in the literature to date. Here, we report our experience of two cases of acute respiratory distress syndrome (ARDS) with DAD following DLI after allo-HSCT, rapidly extending to fatal pulmonary fibrosis. Submission was approved by our institutional review board and informed consent was obtained.

A 68-year-old male patient (patient 1) with acute myeloid leukaemia received haplo-identical allo-HSCT after a thiotepa, fludarabine and busulfan conditioning regimen. No major complications occurred. Prophylactic haplo-DLI was performed at day 140. 4 days after, the patient developed fever and dyspnoea, without any symptoms of GVHD. Chest computed tomography (CT) revealed diffuse bilateral areas of ground-glass attenuation. The patient was admitted early to the intensive care unit (ICU) with isolated acute respiratory failure (ARF). Initial noninvasive infectious workup identified a co-infection with Klebsiella pneumoniae in sputa and human herpesvirus 6 (HHV6) in the nasopharyngeal aspirate. Repeated induced sputa for Pneumocystis jiroveci PCR were negative. All other infectious tests were negative. Cardiac echography was performed to rule out cardiac pulmonary oedema. We started antibiotics, ganciclovir and corticosteroids in light of the radiological appearance. The ventilation strategy consisted of high-flow oxygen and noninvasive ventilation (NIV). Respiratory failure progressed to ARDS [4]. Chest CT abnormalities worsened with extensive ground-glass opacities, traction bronchiectasis and subpleural honeycombing. We initiated empirical voriconazole, stopped it when negative $\beta$-D-glucan result was available and increased corticosteroids to $2 \mathrm{mg} \cdot \mathrm{kg}^{-1}$ per day. Because of the absence of improvement, CT-guided lung biopsy was performed. Histology revealed interstitial fibrosis with associated features compatible with DAD in the organising phase. Tumoural infiltration, GVHD, pulmonary veno-occlusive disease, granuloma and pathogens were excluded in the limit of the small biopsy (figure 1). The patient's respiratory status worsened, as did the chest CT scan fibrosis features, and the patient died after a 35-day ICU stay.

A 68-year-old male patient (patient 2) with myeloma underwent human leukocyte antigen-identical sibling allo-HSCT after a conditioning regimen consisting of fludarabine, thymoglobulin and busulfan. The patient was included in a phase I protocol of infusion of selected donor natural killer (NK) cells, currently under investigation (www.clinicaltrials.gov identifier NCT01853358). NK DLI was performed at day 103 post-allo-HSCT. 10 days later, he developed fever and dyspnoea. He was admitted to the ICU 30 days after DLI, with isolated ARF requiring $10 \mathrm{~L} \cdot \mathrm{min}^{-1}$ oxygen and no symptoms of GVHD. Chest CT revealed diffuse, bilateral, patchy ground-glass opacities with consolidation, traction bronchiectasis and a crazy-paving aspect. Noninvasive infectious work-up identified a co-infection with K. pneumoniae and adenovirus in respiratory samples. Cardiac pulmonary oedema was ruled out. Antibiotics, cidofovir and corticosteroids were initiated. The ventilation strategy consisted of high-flow oxygen and NIV. The patient's respiratory status worsened with ARDS development, requiring mechanical ventilation, prone position, neuromuscular blockade and nitric oxide. Microbiological samples were repeated, identifying Candida tropicalis, Candida albicans and Enterococcus faecium. P. jiroveci PCR was negative. In this context, as we could not distinguish 

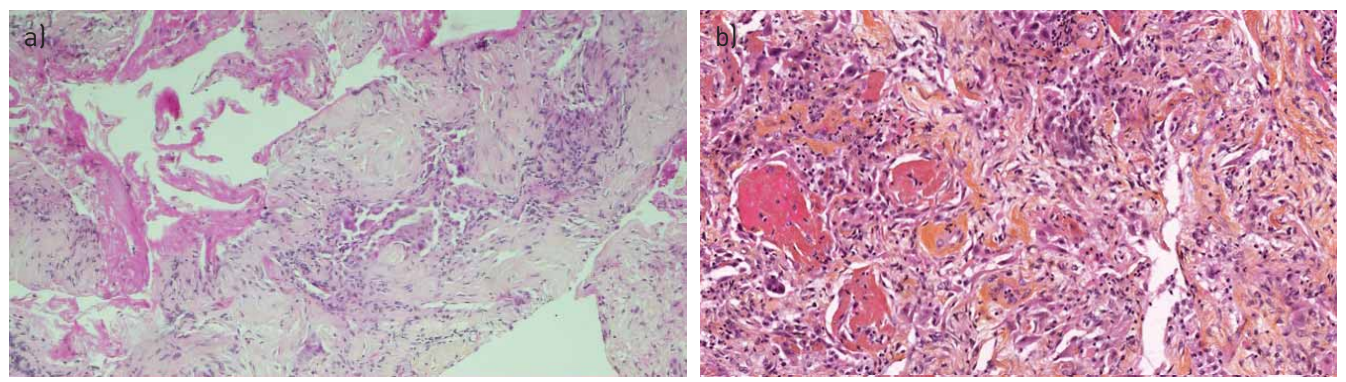

FIGURE 1 Histologic analysis of lung biopsy. a) Computed tomography-guided lung biopsy of patient 1 (haematoxylin-eosin-saffron staining, magnification $\times 100$ ) revealing features compatible with diffuse alveolar damage (DAD) in the organising phase, with interstitial fibrosis. The architecture is fully modified with diffuse interstitial fibrosis, dystrophic pneumocytes, an inflammatory infiltrate of mononuclear cells and intra-alveolar fibrin deposits. b) Post mortem lung biopsy of patient 2 (haematoxylin-eosin staining, magnification $\times 200$ ) revealing $\mathrm{DAD}$ in the subacute organising phase. Alveolar septa are expanded by myxoid-appearing fibrous tissue and pneumocyte hyperplasia is prominent. A residual hyaline membrane is still visible.

infection from colonisation or bronchitis, we started antibiotics. The chest CT abnormalities worsened with fibrosis features apparition. As respiratory status was worsening, corticosteroids were increased to $2 \mathrm{mg} \cdot \mathrm{kg}^{-1}$ per day. The patient died of refractory ARDS after a 31-day ICU stay. Post mortem lung biopsy was performed, revealing DAD in the subacute organising phase (figure 1).

ARDS with DAD following DLI is an unknown complication. Interestingly, our two patients developed the same clinical, radiological and histological picture with similar kinetics. Both were leukopenic, completely asymptomatic before DLI and developed isolated hypoxemic ARF around 7 days after DLI, with no symptoms of GVHD, and had co-infection with bacteria and viruses. CT scanning revealed ground-glass opacities, rapidly progressive to fibrosis. Similarly, histology identified DAD, leading to pulmonary fibrosis. They both died of refractory hypoxaemia.

Pathology revealed DAD, a pathological entity considered as the hallmark of ARDS [5]. It includes several phases evolving toward fibrosis [3]. DAD has been demonstrated as a major risk factor for ARDS mortality in a recent meta-analysis [6].

DLI represents a potential aggression factor, leading to a cytokine storm. NisHIE et al. [7] recently reported a significant elevation of pro-inflammatory cytokines and chemokines in serum after lymphocyte reinfusion. Allo-HSCT patients are frequently infected or colonised by virus in respiratory samples, especially herpesvirus and adenovirus. Recent data suggest the role of virus as a trigger, contributing to lung epithelial injury, promoting pulmonary fibrosis [8]. Moreover, in leuko-lymphopenic patients with ARDS, alveolar macrophages have been shown to be deactivated and ineffective at releasing pro- and anti-inflammatory cytokines [9], which are essential for lung repair [10]. We can assume that DLI may represent a first inflammatory hit, leading to a subsequent lung injury after exposure to a respiratory virus, which could play a major role in fibrosis development. In this context, the lung cannot be repaired, leading to pulmonary fibrosis. The timing demands DLI imputability, as well as the absence of improvement under adapted broad-spectrum antimicrobials, and the exclusion of all other aetiologies. However, we cannot formally exclude the initial role of infection as the first hit, as we documented several pathogens that may be present before DLI. It is important to note that both patients received chemotherapy before allo-HSCT, including treatments such as aracytine or melphalan, both of which have pulmonary toxicity. However, they did not present any respiratory events with the expected timing, making us suspect DLI imputability. Moreover, older studies have suggested that infused lymphocytes lead to lung injury in murine GVHD models, although the lungs have not been generally considered as a classic target of acute GVHD.

ARF after allo-HSCT is frequent and must be treated as an infectious complication, considering the major immunodeficiency of these patients. The diagnostic strategy includes an exhaustive infectious work-up, as the absence of diagnosis is a major prognostic factor, and adapted antimicrobial treatment should be quickly initiated [11, 12]. After ruling out infection, idiopathic pneumonia syndrome (IPS) should be investigated [13]. Current diagnostic criteria for IPS are based on fulfilling two major requirements: 1) widespread alveolar injury with symptoms and signs of pneumonia, and 2) the absence of active lower respiratory tract infection [13]. Interestingly, SEO et al. [14] reported that $57 \%$ of patients with diagnostic IPS had occult infectious aetiologies detected by currently available diagnostic methods, the most frequent pathogens being HHV6 (29\%), rhinovirus (12\%), cytomegalovirus (12\%) and Aspergillus (12\%). Recent data support the use of antiviral treatment in this context. The link between DLI and viral infection is complex. KISHI et al. [15] reported one patient who received DLI while having life-threatening respiratory syncytial 
virus (RSV) pneumonia. His respiratory condition improved after DLI and RSV became undetectable. This illustrates the extreme complexity of the immune response, which can have a positive or negative effect according to the timing and kinetics of anti- or pro-inflammatory cytokine responses.

Thus, in allo-HSCT patients developing severe ARDS following DLI, DAD should be considered. In this context, empirical viral and fungal treatment is questionable, especially when corticosteroids are started. Given the growing use of DLI and the development of novel techniques, such as NK DLI, intensivists will be increasingly asked to manage these patients. Further studies are needed to confirm these data, and define optimal diagnostic and therapeutic strategy.

0

@ERSpublications

Fatal ARDS with diffuse alveolar damage: donor lymphocyte infusion imputability? http://ow.ly/WhxP3034P6s

Colombe Saillard ${ }^{1}$, Magali Bisbal ${ }^{2}$, Antoine Sannini ${ }^{2}$, Laurent Chow-Chine ${ }^{2}$, Jean-Paul Brun ${ }^{2}$, Samia Harbi ${ }^{1}$, Sabine Furst ${ }^{1}$, Didier Blaise ${ }^{1,3}$, Maria Paciencia ${ }^{4}$, Véronique Secq ${ }^{3,5,6}$ and Djamel Mokart ${ }^{2}$

${ }^{1}$ Hematology Dept, Institut Paoli Calmettes, Marseille, France. ${ }^{2}$ Polyvalent Intensive Care Unit, Dept of Anesthesiology and Critical Care, Institut Paoli Calmettes, Marseille, France. ${ }^{3}$ Aix Marseille Université, Marseille, France. ${ }^{4}$ Pathology Dept, Institut Paoli Calmettes, Marseille, France. ${ }^{5}$ Pathology Dept, Hôpital Nord, Marseille, France. ${ }^{6}$ INSERM, U1068 Stress Cellulaire, Marseille, France.

Correspondence: Djamel Mokart, Polyvalent Intensive Care Unit, Department of Anesthesiology and Critical Care, Institut Paoli Calmettes, 232 Boulevard Sainte Marguerite, 13009, Marseille Cedex 09, France.

E-mail: mokartd@ipc.unicancer.fr

Received: June 072016 | Accepted after revision: Aug 022016 | First published online: Sept 012016

Conflict of interest: None declared.

Acknowledgements: C. Saillard and D. Mokart drafted the manuscript. C. Saillard, M. Bisbal, A. Sannini, L. Chow-Chine, J-P. Brun, S. Harbi, S. Furst, D. Blaise and D. Mokart participated in patient care. M. Paciencia and V. Secq carried out the histological analysis. All authors critically revised the manuscript and approved the final submitted version. D. Mokart had full access to all the data and takes responsibility for the integrity of the data.

\section{References}

1 Nikiforow S, Alyea EP. Maximizing GVL in allogeneic transplantation: role of donor lymphocyte infusions. Hematology Am Soc Hematol Educ Program 2014; 2014: 570-575.

2 Chang X, Zang X, Xia CQ. New strategies of DLI in the management of relapse of hematological malignancies after allogeneic hematopoietic SCT. Bone Marrow Transplant 2016; 51: 324-332.

3 Papazian L, Calfee CS, Chiumello D, et al. Diagnostic workup for ARDS patients. Intensive Care Med 2016; 42: 674-685.

4 Ranieri VM, Rubenfeld GD, Thompson BT, et al. Acute respiratory distress syndrome: the Berlin Definition. JAMA 2012; 307: 2526-2533.

5 Lorente JA, Cardinal-Fernandez P, Munoz D, et al. Acute respiratory distress syndrome in patients with and without diffuse alveolar damage: an autopsy study. Intensive Care Med 2015; 41: 1921-1930.

6 Cardinal-Fernandez P, Bajwa EK, Dominguez-Calvo A, et al. The presence of diffuse alveolar damage on open lung biopsy is associated with mortality in patients with acute respiratory distress syndrome: a systematic review and meta-analysis. Chest 2016; 149: 1155-1164.

7 Nishie M, Fujii N, Mimura Y, et al. Vigorous inflammatory responses in noninfectious pulmonary complication induced by donor lymphocyte infusion. Transfusion 2016; 56: 231-236.

8 Pulkkinen V, Salmenkivi K, Kinnula VL, et al. A novel screening method detects herpesviral DNA in the idiopathic pulmonary fibrosis lung. Ann Med 2012; 44: 178-186.

9 Mokart D, Darmon M, Azoulay E. The alveolar macrophage and acute respiratory distress syndrome: a silent actor? Am J Respir Crit Care Med 2014; 189: 499-500.

10 Byrne AJ, Maher TM, Lloyd CM. Pulmonary macrophages: a new therapeutic pathway in fibrosing lung disease? Trends Mol Med 2016; 22: 303-316.

11 Saillard C, Blaise D, Mokart D. Critically ill allogeneic hematopoietic stem cell transplantation patients in the intensive care unit: reappraisal of actual prognosis. Bone Marrow Transplant 2016; 51: 1050-1061.

12 Mokart D, Saillard C, Sannini A, et al. Neutropenic cancer patients with severe sepsis: need for antibiotics in the first hour. Intensive Care Med 2014; 40: 1173-1174.

13 Panoskaltsis-Mortari A, Griese M, Madtes DK, et al. An official American Thoracic Society research statement: noninfectious lung injury after hematopoietic stem cell transplantation: idiopathic pneumonia syndrome. Am J Respir Crit Care Med 2011; 183: 1262-1279.

14 Seo S, Renaud C, Kuypers JM, et al. Idiopathic pneumonia syndrome after hematopoietic cell transplantation: evidence of occult infectious etiologies. Blood 2015; 125: 3789-3797.

15 Kishi Y, Kami M, Oki Y, et al. Donor lymphocyte infusion for treatment of life-threatening respiratory syncytial virus infection following bone marrow transplantation. Bone Marrow Transplant 2000; 26: 573-576. 